\title{
Misexpression of the Caenorhabditis elegans miRNA let-7 Is Sufficient to Drive Developmental Programs
}

\author{
G.D. HAYES AND G. RUVKun \\ Department of Molecular Biology, Massachusetts General Hospital and Department of Genetics, \\ Harvard Medical School, Boston, Massachusetts 02114
}

\begin{abstract}
The Caenorhabditis elegans microRNAs (miRNAs) lin-4 and let-7 promote transitions between stage-specific events in development by down-regulating the translation of their target genes. Expression of let-7 is required at the fourth larval stage for the proper transition from larval to differentiated, adult fates in the hypodermis; however, it was not known whether expression of let-7 is sufficient to specify these adult fates. To test this, we created fusion genes between lin-4 and let-7 that direct the expression of let-7 two stages early, at the L2 stage. We find that animals bearing the fusion genes show precocious adult development at the L4 stage, indicating that temporal misexpression of let-7 is sufficient to direct the larval-to-adult transition. Additionally, an RNA interference (RNAi)-based screen for enhancers of the precocious phenotype identified the period ortholog lin-42, among other genes, which are candidate modulators of the effects of let-7 expression. let- 7 is conserved throughout bilaterian phylogeny, and orthologs of its targets have roles in vertebrate development, suggesting the importance of understanding how let-7 promotes terminal differentiation in C. elegans and other organisms.
\end{abstract}

The first two miRNAs discovered, lin-4 and let-7, regulate the timing of certain developmental events that occur in particular stages of $C$. elegans development. lin-4 is required for the transition from L1 to L2 and from L2 to L3 developmental programs in the hypodermal seam cells, among other tissues. Animals lacking lin-4 reiterate in each larval stage and, at the nominal adult stage, seam cell division patterns that are normally confined to the L1 stage (Chalfie et al. 1981; Ambros and Horvitz 1984). lin-4 promotes the normal progression through developmental programs by down-regulating translation of its target genes, lin-14 and lin-28 (Lee et al. 1993; Wightman et al. 1993; Moss et al. 1997). Inactivation of the lin-4 targets causes the opposite heterochronic phenotype as loss of lin-4: Developmental programs appropriate to later stages occur precociously.

Similarly, let-7 acts to trigger a transition from larval to adult patterns of development. In let-7 mutants, the hypodermal seam cells continue to divide in adults, rather than fusing and secreting the adult-specific cuticular structure known as alae (Reinhart et al. 2000). let-7 acts by inhibiting the translation of its targets lin-4l and $h b l-1$ (Slack et al. 2000; Abrahante et al. 2003; Lin et al. 2003), and downregulation of these targets in turn allows synthesis of the transcription factor encoded by lin-29 (Rougvie and Ambros 1995), the most downstream gene discovered in the heterochronic pathway, the set of miRNAs and their protein-coding targets that regulate developmental timing (Ambros and Horvitz 1984). At the larval-to-adult transition, LIN-29 directs expression of genes (such as collagens) involved in synthesis of the adult cuticle and inhibits transcription of those required for synthesis of larval cuticle (Liu et al. 1995; Rougvie and Ambros 1995). Recently, let-7 was shown to target additional genes at the larval-toadult transition, including the gene encoding the nuclear hormone receptor DAF-12 as well as several other transcription factors (Grosshans et al. 2005).
Although a number of other genes that regulate developmental timing in C. elegans have been identified, it remains unclear how transitions between developmental programs following the L1 stage are promoted. Work on the let-7 paralogs mir-48, mir-84, and mir-241 has suggested that they have a role in the transition to L3 developmental programs (Abbott et al. 2005; Hayes 2005; Li et al. 2005). It was not clear whether any outputs of the heterochronic pathway that are independent of let-7 expression are required to promote the larval-to-adult transition.

Understanding how let-7 promotes the transition to terminal differentiation in C. elegans is important because of the roles that let-7 has in many animals. let- 7 is perfectly conserved throughout bilaterian phylogeny, and roles for its target, lin-41, has also been identified in vertebrate development (Pasquinelli et al. 2000; Kloosterman et al. 2004; Lancman et al. 2005; Schulman et al. 2005). Analogously to its role in C. elegans, up-regulation of let-7 is associated with transition from the larval to pupal stage in Drosophila (Sempere et al. 2002, 2003; Bashirullah et al. 2003). Reduced expression of let-7 has also been observed in a variety of tumors, suggesting it may have a broader role in promoting terminal differentiation (Takamizawa et al. 2004; Johnson et al. 2005; Karube et al. 2005).

Here, we show that precocious expression of let-7 under the control of the lin-4 promoter is sufficient to drive premature execution of adult developmental programs. At the fourth larval stage, animals carrying a lin-4::let-7 fusion gene show precocious adult differentiation of the hypodermis that is dependent on lin-29 activity. We also report results of an RNAi-based screen to identify enhancers of the precocious phenotype that results from early let-7 expression. Inactivation of certain genes involved in developmental timing and RNA metabolism causes an enhanced precocious phenotype in animals bearing the lin-4::let-7 fusion gene. 


\section{HAYES AND RUVKUN}

\section{SECTION THEMES miRNA Fusion Genes}

To address whether expression of the miRNA let-7 is sufficient to direct the larval-to-adult transition in the lateral hypodermal seam cells of C. elegans, we expressed let-7 in the first larval stage, two stages earlier than it is normally expressed. We designed two constructs, both placing the let-7 precursor and downstream sequence immediately $3^{\prime}$ to the putative lin-4 promoter and precursor (Fig. 1A). We chose to use the lin-4 promoter because mutations in lin-4 and let-7 affect the same tissues, suggesting that they are expressed in the same places. In addition, although it has now been shown that miRNA-coding genes are transcribed by RNA polymerase II (Cai et al. 2004; Lee et al. 2004), it was not originally clear how
miRNA expression is controlled. We included the lin-4 precursor because of the possibility that elements regulating lin-4 transcription could be embedded in the precursor sequence. Construct A (ExA) included 573 nucleotides upstream of the lin-4 precursor, which was shown to be sufficient to rescue a lin-4 mutant (Lee et al. 1993). Construct B $(\operatorname{ExB})$ was nearly identical, except that it included 1668 nucleotides upstream of the lin-4 precursor, and the sequence $3^{\prime}$ of the lin-4 precursor was extended by 8 nucleotides before the fusion point to let-7.

\section{The lin-4 Promoter Can Drive Precocious Expression of let-7}

Northern blot analysis demonstrated that both constructs Ex[lin-4A::let-7] (data not shown) and Ex[lin-
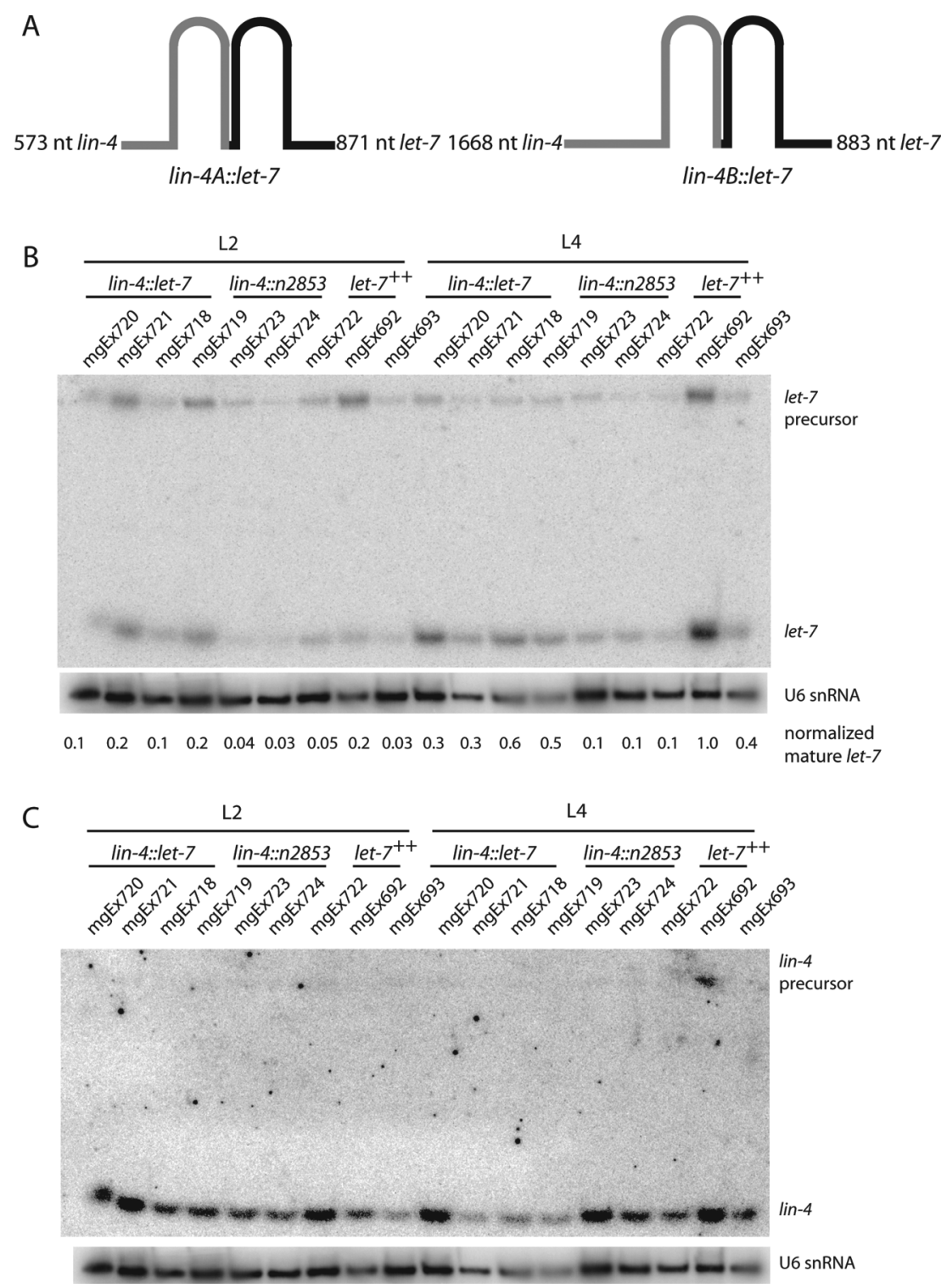

Figure 1. Fusions to lin-4 sequence drive precocious expression of let-7. (A) Schematics of the lin-4A::let-7 and lin-4B::let-7 fusion constructs. $(B)$ Blot of RNA isolated from lin-4B::let-7 and control lines at the L2 and L4 stages probed for let-7. Information on the transgenic arrays is presented in Table 1. (C) The same blot reprobed for lin-4. 
$4 B::$ let-7] (Fig. 1B) express the mature let-7 miRNA by the beginning of the L2 stage. This was also the case with Ex[lin-4::let-7(n2853)], in which the $n 2853$ point mutation was included. Surprisingly, when let-7 was overexpressed under its own promoter from a 2459-bp genomic fragment beginning 1576 nucleotides $5^{\prime}$ of the let-7 precursor, it was also detected by early L2. The method used here normally reveals let-7 expression at a low level in the L3 stage in wild-type C. elegans (Reinhart et al. 2000), although a more sensitive method has detected low-level expression at the L2 stage (Abbott et al. 2005). It is possible that a negative regulatory element was not included in the let-7 overexpression construct or that increased expression from the multicopy array reveals let-7 earlier than is the case when it is expressed from the endogenous let-7 locus. The temporal response element described by Johnson et al. (2003) as necessary for proper regulation of let-7 was included. Quantitation of the signal from the 22nucleotide band in each lane and normalization of loading relative to U6 small nuclear RNA (snRNA) signal revealed that let-7 was expressed at similar levels in the L2 stage in all lines, although the highest level of expression was achieved under the lin-4 promoter (Fig. 1B). The apparently slightly lower expression level of the $n 2853$ control construct could be due to imperfect hybridization between the probe and the mutated target sequence. In all cases, the abundance of the mature form of the let-7 miRNA increased at the L4 stage. Interestingly, in each line, an accumulation of the precursor form of let-7 relative to the abundance of the mature product was observed in the L2, but this accumulation was resolved by the L4 stage (Fig. 1B and data not shown). This suggests the possibility that processing of let-7 by the Dicer-ALG-1/2 complex (Grishok et al. 2001; Hutvagner et al. 2001), as well as the transcription of let-7, could be temporally regulated. No similar L2-stage accumulation of lin-4 precursor was observed, demonstrating that miRNA processing in general was not disrupted (Fig. 1C).

Both of the lin-4::let-7 fusion constructs drove production of functional let-7, as demonstrated by their ability to rescue a let-7 null mutant (Table 1). However, all of the lines that were tested showed at most weak rescue of a lin4 null (data not shown). In addition, when RNA derived from animals carrying the lin-4B::let-7 transgenes $m g E x 719$ and $m g E x 720$ in a lin-4 null background was probed, no clear lin-4 expression was detected (data not shown). These observations suggest that the lin-4 precursor produced from these fusion genes may be degraded rather than processed to mature lin-4. Many miRNAs are found in genomic clusters (such as mir-35-mir-41 in C. elegans and let-7 and the lin-4 homolog, miR-125, in Drosophila) and are processed from a single primary transcript (Lau et al.

Table 1. Phenotypes Generated by let-7 Precocious- and Overexpression Transgenes

\begin{tabular}{|c|c|c|c|c|}
\hline Strain/Array & Construct & $\begin{array}{c}\text { Injection } \\
\text { concentration } \\
(\mathrm{ng} / \mu \mathrm{l})\end{array}$ & $\begin{array}{c}\text { Precocious alae } \\
\text { at L4 stage } \\
(\%[\mathrm{n}])\end{array}$ & $\begin{array}{c}\text { Rescue of } \\
\text { let- } 7 \\
(\%[\mathrm{n}]) \\
\end{array}$ \\
\hline$m g E x 704$ & lin-4A::let-7 & 40 & $0(5)$ & n.d. \\
\hline$m g E x 705$ & lin-4A::let-7 & 40 & n.d. & $20(10)$ \\
\hline$m g E x 706$ & lin- $4 A \because:$ let-7 & 20 & n.d. & $77(22)$ \\
\hline$m g E x 707$ & lin-4A::let-7 & 20 & n.d. & $80(10)$ \\
\hline$m g E x 708$ & lin- $4 A::$ let -7 & 20 & n.d. & $100(26)$ \\
\hline$m g E x 709$ & lin-4A::let-7 & 20 & $6(17)$ & $71(14)$ \\
\hline $\operatorname{mgEx} 710$ & lin-4A::let-7 & 40 & $0(7)$ & $<5(>20)$ \\
\hline$m g E x 711$ & lin-4B::let-7 & 15 & $80(5)$ & n.d. \\
\hline$m g E x 712$ & lin-4B::let-7 & 15 & $33(12)$ & n.d. \\
\hline$m g E x 713$ & lin-4B::let-7 & 15 & $40(10)$ & $40(10)$ \\
\hline$m g E x 714$ & lin-4B::let-7 & 10 & $0(3)$ & $59(12)$ \\
\hline $\operatorname{mgEx} 715$ & lin- $4 B::$ let-7 & 10 & $29(7)^{b}$ & $80(5)$ \\
\hline $\operatorname{mgEx} 716$ & lin-4B::let -7 & 10 & $69(26)$ & n.d. \\
\hline $\operatorname{mgEx} 717^{\mathrm{a}}$ & lin-4B::let -7 & 5 & $0(7)$ & $100(9)$ \\
\hline $\operatorname{mgEx} 718^{\mathrm{a}}$ & lin-4B::let-7 & 5 & $35(17)$ & n.d. \\
\hline $\operatorname{mgEx} 719^{\mathrm{a}}$ & lin-4B::let-7 & 5 & $43(7)$ & n.d. \\
\hline$m g E x 720^{\mathrm{a}}$ & lin- $4 B::$ let -7 & 1 & $38(8)$ & $100(6)$ \\
\hline $\operatorname{mgEx} 721^{\mathrm{a}}$ & lin- $4 B::$ let-7 & 1 & $45(31)$ & n.d. $=$ \\
\hline $\operatorname{mgEx} 722^{\mathrm{a}}$ & $\operatorname{lin}-4 B:: n 2853$ & 5 & $0(12)$ & n.d. $=$ \\
\hline $\operatorname{mgEx} 723^{\mathrm{a}}$ & $\operatorname{lin}-4 B:: n 2853$ & 1 & $0(20)$ & n.d. $=$ \\
\hline $\operatorname{mgEx} 724^{\mathrm{a}}$ & $\operatorname{lin}-4 B:: n 2853$ & 1 & $4(24)$ & $0(9)$ \\
\hline$m g E x 692^{\mathrm{a}}$ & let-7++ & 1 & $0(14)$ & $100(8)$ \\
\hline$m g E x 693^{\mathrm{a}}$ & let-7++ & 1 & $0(16)$ & n.d. $=$ \\
\hline lin-41(ma104) & n.a. & n.a. & $50(12)$ & n.d. \\
\hline$m g \operatorname{Ex} 719^{a}$ & lin-4B::let-7 & 5 & $78(9)$ & n.d. \\
\hline$m g E x 719$ & & & & \\
\hline $\operatorname{lin}-29(R N A i)^{\mathrm{a}}$ & lin- $4 B::$ let -7 & 5 & $0(9)$ & n.d. \\
\hline$m g E x 720$; vector $(R N A i)$ & lin-4B::let-7 & 1 & $26(19)^{b}$ & n.d. \\
\hline $\begin{array}{l}\text { mgEx720; } \\
\text { lin-29(RNAi) }\end{array}$ & lin-4B::let-7 & 1 & $0(14)^{\mathrm{b}}$ & n.d. \\
\hline
\end{tabular}

${ }^{a}$ All observations reported on this line of the table were done in a $w I s 54[s c m:: g f p] \mathrm{V} ;$ let-7(mn112) unc-3(e151) X background.

${ }^{\mathrm{b}}$ Scored in a let-7(mn112) unc-3(e151) background.

$=$ This transgene rescued let $-7(\mathrm{mn} 112)$ (only at $15^{\circ} \mathrm{C}$ for lin-4::n2853 lines), but the penetrance of rescue was not determined. n.d. indicates not determined; n.a. indicates not applicable. 
2001; Bashirullah et al. 2003). However, the mature lin-4 and let-7 sequences are positioned more closely (7-15-bp separation) in our fusion genes than the miRNAs in these naturally occurring clusters ( $>30$-bp separation).

\section{Early Expression of let-7 Generates Precocious Development}

It was expected that early expression of let-7 would cause precocious down-regulation of its target, LIN-41, causing a phenotype similar to that seen in animals bearing loss-of-function mutations in lin-41. Animals lacking LIN-41 are dumpy (Dpy) and partly sterile. In wild-type C. elegans, the lateral hypodermal seam cells fuse and secrete a cuticular structure known as alae at the L4-toadult molt (Sulston and Horvitz 1977). In lin-41 loss-offunction mutants, both seam cell fusion and production of alae occur one stage early, at the L3-to-L4 molt (Slack et al. 2000). Animals expressing both Ex[lin-4A::let-7] and Ex[lin-4B::let-7] were Dpy and had a partially penetrant egg-laying defect (data not shown). However, animals expressing Ex[lin-4A::let-7] only very rarely produced alae precociously, whereas those expressing Ex [lin-4B:: let-7] produced alae with a penetrance similar to that of lin-41(ma104); 29-80\% of animals bearing Ex [lin-4B:: let-7] and $50 \%$ of lin- 41 mutants produced alae covering at least one of the lateral hypodermal seam cells at the L3to-L4 molt (Table 1). As shown in Figure 2, both lin-41(ma104) animals and those expressing Ex [lin-4B:: let-7] often produced "patchy" alae during the L4 stage, with some seam cells precociously expressing the adult fate, while others continued to express the larval fate. The "patchy alae" phenotype has also been observed for other heterochronic mutants (Ambros 1989).

Consistent with the results for alae formation, animals bearing lin-4B::let-7 transgenes showed precocious expression of a reporter for col-19, which encodes a collagen that is a component of the adult cuticle. col-19, which is positively regulated by LIN-29, is expressed only in the adult stage in wild-type C. elegans (Abrahante et al. 1998); $75 \%(n=12)$ of animals carrying $m g E x 719$ [lin-4B::let-7] expressed the veIs 13 [col-19::gfp] reporter in the L4 stage. Animals bearing lin-4B::let-7 transgenes only rarely expressed col-19::gfp as early as the L3 stage (Fig. 3).

\section{Precocious Alae Result from Early Expression of let-7}

To test whether the precocious phenotype generated by the Ex[lin-4B::let-7] transgenes was due to expression of let-7, we introduced the $n 2853$ point mutation into the lin-4B::let-7 construct. Introduction of the $n 2853$ mutation was expected to block let-7 function, because let-7(n2853) mutant worms have a temperature-sensitive lethal phenotype similar to that of let-7(mn112) null mutants. In fact, animals bearing the Ex [lin-4B:: let-7(n2853)] constructs only very rarely $(0-4 \%)$ produced alae precociously (Table 1$)$. Those animals bearing $m g E x$ [lin-4B::let-7(n2853)] that did produce alae precociously produced only faint alae, not distinct patches as observed with animals bearing the Ex[lin-4B::let-7]

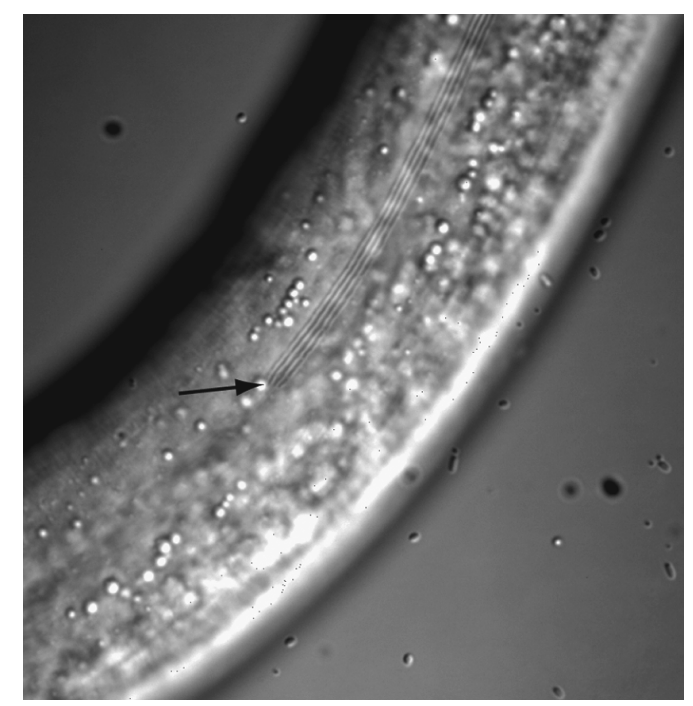

Figure 2. A typical L4 larva bearing the lin-4B::let-7 transgene showing precocious alae formation. This animal expressed a mix of larval and adult fates in the hypodermis, indicated by the break in the alae (arrow).

transgenes (data not shown). The fact that the introduction of the $n 2853$ point mutation abrogates the precocious phenotype induced by expression of Ex [lin-4B::let-7] demonstrates that the precocious phenotype results from early expression of let-7, not overexpression of lin-4.

The precocious phenotype generated by the lin-4::let-7 constructs was not a product of simple overexpression of let-7. Animals bearing the Ex[let- $\left.7^{++}\right]$transgenes never formed alae precociously (Table 1), even though they produced let-7 at the L 2 stage at a level comparable to that of animals bearing the lin-4::let-7 constructs (Fig. 1B).

\section{The Heterochronic Pathway Mediates the Effects of Precocious let-7 Expression through lin-29}

To confirm that the precocious phenotype produced by expression of Ex[lin-4B::let-7] is mediated by the established heterochronic pathway, we inactivated the terminal gene in this pathway, lin-29, by feeding RNAi. Animals bearing either of two lin-4B::let-7 transgenes produced alae at the L3-to-L4 molt when fed vector RNAi, but they never produced alae when fed lin-29(RNAi) (Table 1), indicating that the precocious phenotype produced by expression of the lin-4::let-7 fusion gene is mediated by lin-29.

\section{RNAi Screen for Enhancers of the Precocious lin-4::let-7 Phenotype}

Although the lin- $4 B::$ let-7 construct drives precocious production of let-7 two stages early, by the beginning of the L2 stage, its expression results in production of alae and expression of col-19::gfp only one stage early, at the L3-to-L4 molt. It seemed possible that some factor might inhibit the early action of let-7 or that some other factor necessary for let-7 function might not normally be present as early as the L2 stage. 
To identify such factors, we conducted a feeding RNAi screen using a library of 438 clones selected because they were expected to have a role in RNA metabolism, RNAi, genome stability and transposon silencing, miRNA function, or control of developmental timing. Animals bearing $m g E x 719$ [lin-4B::let-7] in a col-19::gfp; let-7(mn112) unc-3(e151) background were fed each clone, and they and their progeny were scored for expression of col-19:: $g f p$ earlier than the L4 stage; 128 clones emerged that either appeared to cause expression of col-19::gfp earlier than the L4 stage or that caused other phenotypes (such as sickness or sterility) specifically in the animals bearing the lin-4::let-7 transgene (data not shown). None of the seven vector clones included and scored blindly was noted to cause precocious col-19::gfp expression nor any other phenotypes in larvae. 25 of the 128 clones appeared to enhance precocious expression of col-19::gfp in animals bearing the lin-4::let-7 transgene upon a single retest (Table 2; data not shown). Four of the 25 positive clones that caused the most potent or penetrant precocious expression of col-19::gfp were examined by Nomarski microscopy to determine the age of the green animals. The clones corresponding to lin-42 (period), Y57G11A.5 (which encodes a predicted RNA-binding protein that may be involved in splicing), and Y77E11A.7 and T26A5.5, which are required for genome stability, were all confirmed to cause col-19::gfp expression by the L3 stage in animals bearing $m g E x 719$ [lin-4B::let-7] (Fig. 3A; Table 2).

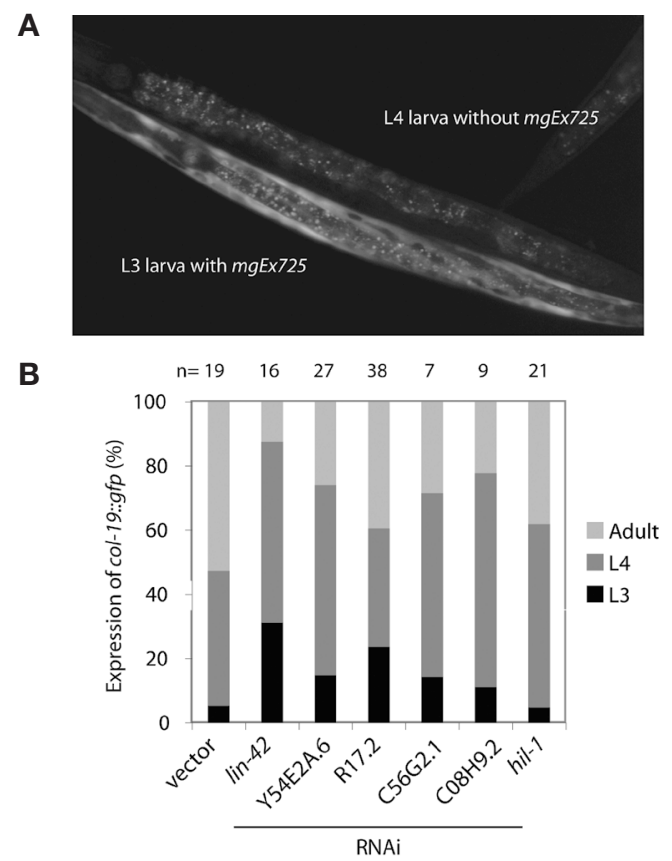

Figure 3. Enhancement of the $m g E x 725$ [lin-4B::let-7] precocious col-19::gfp expression phenotype by RNAi. (A) lin42(RNAi) enhanced the precocious col-19::gfp expression phenotype of animals bearing the lin- $4 B::$ let -7 transgene. On the bottom is an L3 animal bearing $m g E x 725$ [lin-4B::let-7] and expressing col-19::gfp precociously. Above it is an L4 animal without the $m g \operatorname{Ex} 725$ transgene and showing only background fluorescence. $(B)$ Effect of feeding RNAi against the indicated clone on the age distribution of animals expressing col-19::gfp in the $\mathrm{F}_{1}$ generation.
Table 2. Possible Enhancers of the Precocious col-19::gfp Expression in Animals Bearing mgEx719[lin-4B::let-7]

\begin{tabular}{cc}
\hline Developmental timing & Nucleases \\
F47F6.2 (lin-42) & R17.2 \\
F11A1.3 (daf-12) & Y39G8C.1 (xrn-1) \\
Argonaute orthologs & F31E3.4 \\
F55A12.1 & C50C3.7 \\
C35D6.3 & ZK1098.3 \\
Nucleic acid binding & Helicases \\
C08H9.2 & Y54E2A.6 \\
C56G2.1 & Genome stability / \\
C44E4.4 & transgene silencing \\
R05H10.2 & R06C7.7 (lin-61) \\
M4.2 (puf-4) & T26A5.5 \\
Y57G11A.5 & Y77E11A.7 \\
Y116A8C.32 & AC8.1 (pme-6) \\
C30G7.1 (hil-1) & Unknown function \\
mRNA nuclear export & C28A5.1 \\
F10G8.3 (npp-17) & Y54E2A.7 \\
\hline
\end{tabular}

The 25 apparent enhancers were tested a final time with a strain bearing a red fluorescent protein ( $r f p)$-marked lin-4B::let-7 transgene that eliminated background green fluorescence from the original tub-1::gfp-marked lin- $4 B::$ let-7 transgene. Animals bearing the lin-4B::let-7 transgene showed col-19::gfp expression by the L3 stage when grown in synchrony and fed RNAi against the following genes beginning at the L1 stage: lin-42, 7 of 22; C08H9.2, 3 of 23; C56G2.1, 3 of 27; hil-1, 4 of 29; R17.2, 2 of 22; Y54E2A.6, 2 of 18; Y54E2A.7, 3 of 18; lin-61, 6 of 21. No animals lacking the lin-4B::let-7 transgene showed expression of col-19::gfp before the L4 stage. One of 20 lin-4B::let-7 transgene-bearing animals fed vector RNAi dimly expressed col-19::gfp at the L3 stage. For clones that induced relatively penetrant precocious expression of col-19::gfp in the $\mathrm{P}_{0}$ generation, the $\mathrm{F}_{1}$ progeny were washed from the well, and the age of col-19::gfp-expressing animals that bore the lin-4::let-7 transgene was determined (Fig. 3B). Inactivation of lin-42 (period) and R17.2 (which encodes a nuclease) in particular increased the proportion of L3 animals that expressed col-19::gfp as compared to vector. Targeting of Y54E2A.6 (which encodes a helicase) and $\mathrm{C} 08 \mathrm{H} 9.2$ (which encodes a $\mathrm{KH}$-domain protein) had less effect at the L3 stage, but increased the proportion of animals expressing col-19::gfp before the adult stage.

A final category of clone that emerged from the screen was those that allowed let-7 null animals to live in the absence of the lin-4::let-7 transgene. Among the six suppressors was lin-41, a known suppressor of let-7 and one of its established targets (Slack et al. 2000). Three of the six clones (those targeting F55F8.3, K07C5.4, and F17C11.9) corresponded to genes required for assembly of the ribosome or translation. The significance of this finding is unclear, but it may be that the lethality of let-7 derives from derepression of the translation of lin-41 or other let-7 targets and that this lethality is prevented by defects in translation. Two additional suppressors were crn-1, which encodes an endonuclease (Parrish et al. 2003), and puf-3, which encodes an ortholog of Pumilio, an RNA-binding protein implicated in posttranscriptional regulation (Wickens et al. 2002). 


\section{CONCLUSIONS}

The data presented here demonstrate that early expression of the let-7 miRNA is sufficient to drive adult differentiation of the hypodermis and that this precocious phenotype is mediated by the heterochronic gene lin-29. It is perhaps surprising that animals bearing the Ex $\left[\right.$ let $\left.-7^{++}\right]$transgenes failed to produce alae precociously, given that animals expressing both Ex[lin-4B::let-7] and Ex[let- $\left.7^{++}\right]$produced similar levels of mature let- 7 miRNA by the L 2 stage. One explanation is that the lin-4 and let-7 promoters may drive let-7 expression in different tissues and that expression of let-7 in the L2 stage in tissues where the lin-4 promoter is active is required to yield the precocious phenotype. It could be that some factor prevents the early activity of let-7 in the tissues where it is expressed under its own promoter but that this factor is not present in additional tissues where Ex[lin-4B::let-7] drives let-7 expression. Candidates for such a factor include molecules such as LIN-42 and others identified in the enhancer screen.

The period homolog lin-42 has been previously shown to cause precocious development of the hypodermis when it is inactivated (Jeon et al. 1999). Loss of other genes that cause precocious heterochronic phenotypes, such as lin-28, has been shown to up-regulate levels of let-7 (Johnson et al. 2003). It seemed possible that the precocious phenotype of lin-42 mutants could also depend on such up-regulation of let-7. However, the fact that inactivation of lin-42 strongly enhances the precocious phenotype of animals bearing lin-4B::let-7 transgenes suggests that up-regulation of let-7 is not primarily responsible for the precocious phenotype of lin-42 mutants.

It was recently shown that the abundance of mature let-7 and many other miRNAs in vertebrates is controlled at the level of processing of the primary transcript by Drosha (Thomson et al. 2006). No similar regulation has been shown in C. elegans; indeed, transcription of a let-7 reporter (Johnson et al. 2003) and processing of the primary transcript (Bracht et al. 2004) are closely correlated with the appearance of mature let-7. However, regulation of processing, at the level of either the primary transcript or the hairpin precursor, is one reason the precocious phenotypes generated by let-7 expression under the lin-4 promoter could be weaker than anticipated. Indeed, the accumulation of the hairpin precursor form of let-7 at the L2 stage (Fig. 1B) suggested that such regulation might occur.

Additional candidates for factors that could act together with let-7 to direct the larval-to-adult transition were the let-7 paralogs mir-48, mir-84, and mir-241 (Lau et al. 2001; Lim et al. 2003). Indeed, let-7 and mir-84 act together to promote cessation of molting in adults, and overexpression of mir- 84 causes precocious execution of the L3-stage pattern of seam cell divisions in the L2 stage (Hayes 2005). However, when lin-4::mir-48 and lin$4::$ mir- 84 constructs analogous to the lin- $4 B::$ let -7 construct were injected, they caused no obvious precocious phenotypes (data not shown). Animals injected with combinations of these constructs and the lin-4B::let-7 construct showed precocious development, but not to a greater extent than animals bearing Ex[lin-4B::let-7] alone (data not shown). Similarly, no effect on the L2-stage seam cell divisions was noted for the lin-4B::let-7 transgenes, even in combination with the enhancer clones identified here (data not shown). These results suggest that despite their sequence similarity, let-7 and its paralogs have at least partly distinct targets in promoting developmental progression. This finding is in agreement with the observation that the effects of mir- 84 overexpression are similar to but distinct from those of mir-48 overexpression (Hayes 2005; Li et al. 2005) and also with the observation by Leaman et al. (2005) that inactivation of individual miR-310 family members caused similar, but not identical, phenotypes in Drosophila larval development.

The results of the RNAi screen for enhancers of the precocious phenotype caused by expression of lin-4B::let-7 point to possible roles in the regulation of let-7 (at least as expressed from the fusion transgene) for molecules implicated in various aspects of RNA metabolism. One caveat is that the verification of these observations remains incomplete. Retesting with the $r f p$-marked lin- $4: \because$ let-7 transgene failed to detect precocious col-19::gfp expression in the $\mathrm{P}_{0}$ generation of animals for many of the clones. Greater penetrance of precocious phenotypes is expected upon screening of the $\mathrm{F}_{1}$ progeny of animals fed each clone. Determining whether these clones enhance precocious col-19::gfp expression in heterochronic mutant backgrounds (such as lin-14, lin-28, lin-42, and $h b l-1)$ will point to roles the corresponding genes might have in the heterochronic pathway. It would also help clarify the relationship of the enhancers to the regulation of endogenous let-7.

\section{ACKNOWLEDGMENTS}

John Kim and Sylvia Fischer generously shared the sublibrary of RNAi clones they assembled from the library created in Julie Ahringer's laboratory. col-19::gfp was a kind gift from Ann Rougvie. The assistance of Jinling $\mathrm{Xu}$, Snjezana Joksimovic, and Li Xue with microinjections is gratefully acknowledged. Sylvia Lee, Ho Yi Mak, Amy Pasquinelli, Brenda Reinhart, and other members of the Ruvkun lab all contributed advice and helpful discussion throughout the project. This work was supported by National Institutes of Health grant GM44619 to G.R.

\section{REFERENCES}

Abbott A.L., Alvarez-Saavedra E., Miska E.A., Lau N.C., Bartel D.P., Horvitz H.R., and Ambros V. 2005. The let-7 microRNA family members mir-48, mir-84, and mir-241 function together to regulate developmental timing in Caenorhabditis elegans. Dev. Cell 9: 403.

Abrahante J.E., Miller E.A., and Rougvie A.E. 1998. Identification of heterochronic mutants in Caenorhabditis elegans. Temporal misexpression of a collagen::green fluorescent protein fusion gene. Genetics 149: 1335.

Abrahante J.E., Daul A.L., Li M., Volk M.L., Tennessen J.M., Miller E.A., and Rougvie A.E. 2003. The Caenorhabditis elegans hunchback-like gene lin-57/hbl-1 controls developmental time and is regulated by microRNAs. Dev. Cell 4: 625 .

Ambros V. 1989. A hierarchy of regulatory genes controls a larvato-adult developmental switch in C. elegans. Cell 57: 49.

Ambros V. and Horvitz H.R. 1984. Heterochronic mutants of the 
nematode Caenorhabditis elegans. Science 226: 409.

Bashirullah A., Pasquinelli A.E., Kiger A.A., Perrimon N., Ruvkun G., and Thummel C.S. 2003. Coordinate regulation of small temporal RNAs at the onset of Drosophila metamorphosis. Dev. Biol 259: 1

Bracht J., Hunter S., Eachus R., Weeks P., and Pasquinelli A.E. 2004. Trans-splicing and polyadenylation of let-7 microRNA primary transcripts. $R N A$ 10: 1586 .

Cai X., Hagedorn C.H., and Cullen B.R. 2004. Human microRNAs are processed from capped, polyadenylated transcripts that can also function as mRNAs. RNA 10: 1957.

Chalfie M., Horvitz H.R., and Sulston J.E. 1981. Mutations that lead to reiterations in the cell lineages of C. elegans. Cell 24: 59

Grishok A., Pasquinelli A.E., Conte D., Li N., Parrish S., Ha I., Baillie D.L., Fire A., Ruvkun G., and Mello C.C. 2001. Genes and mechanisms related to RNA interference regulate expression of the small temporal RNAs that control $C$. elegans developmental timing. Cell 106: 23.

Grosshans H., Johnson T., Reinert K.L., Gerstein M., and Slack F.J. 2005. The temporal patterning microRNA let-7 regulates several transcription factors at the larval to adult transition in C. elegans. Dev. Cell 8: 321 .

Hayes G.D. 2005. "Control of developmental timing by microRNAs in C. elegans." Ph.D. thesis, Harvard University, Cambridge, Massachusetts.

Hutvagner G., McLachlan J., Pasquinelli A.E., Balint E., Tuschl T., and Zamore P.D. 2001. A cellular function for the RNAinterference enzyme Dicer in the maturation of the let-7 small temporal RNA. Science 293: 834.

Jeon M., Gardner H.F., Miller E.A., Deshler J., and Rougvie A.E. 1999. Similarity of the C. elegans developmental timing protein LIN-42 to circadian rhythm proteins. Science 286: 1141.

Johnson S.M., Lin S.Y., and Slack F.J. 2003. The time of appearance of the $C$. elegans let-7 microRNA is transcriptionally controlled utilizing a temporal regulatory element in its promoter. Dev. Biol. 259: 364

Johnson S.M., Grosshans H., Shingara J., Byrom M., Jarvis R., Cheng A., Labourier E., Reinert K., Brown D., and Slack F.J. 2005. RAS is regulated by the let-7 microRNA family. Cell 120: 635 .

Karube Y., Tanaka H., Osada H., Tomida S., Tatematsu Y., Yanagisawa K., Yatabe Y., Takamizawa J., Miyoshi S., Mitsudomi T., and Takahashi T. 2005. Reduced expression of Dicer associated with poor prognosis in lung cancer patients. Cancer Sci. 96: 111.

Kloosterman W.P., Wienholds E., Ketting R.F., and Plasterk R.H. 2004. Substrate requirements for let-7 function in the developing zebrafish embryo. Nucleic Acids Res. 32: 6284.

Lancman J.J., Caruccio N.C., Harfe B.D., Pasquinelli A.E., Schageman J.J., Pertsemlidis A., and Fallon J.F. 2005. Analysis of the regulation of lin-41 during chick and mouse limb development. Dev. Dyn. 234: 948.

Lau N.C., Lim L.P., Weinstein E.G., and Bartel D.P. 2001. An abundant class of tiny RNAs with probable regulatory roles in Caenorhabditis elegans. Science 294: 858.

Leaman D., Chen P.Y., Fak J., Yalcin A., Pearce M., Unnerstall U., Marks D.S., Sander C., Tuschl T., and Gaul U. 2005. Antisense-mediated depletion reveals essential and specific functions of microRNAs in Drosophila development. Cell 121: 1097

Lee R.C., Feinbaum R.L., and Ambros V. 1993. The C. elegans heterochronic gene lin-4 encodes small RNAs with antisense complementarity to lin-14. Cell 75: 843.

Lee Y., Kim M., Han J., Yeom K.H., Lee S., Baek S.H., and Kim V.N. 2004. MicroRNA genes are transcribed by RNA polymerase II. EMBO J. 23: 4051.
Li M., Jones-Rhoades M.W., Lau N.C., Bartel D.P., and Rougvie A.E. 2005. Regulatory mutations of mir-48, a C. elegans let-7 family microRNA, cause developmental timing defects. Dev. Cell 9: 415 .

Lim L.P., Lau N.C., Weinstein E.G., Abdelhakim A., Yekta S., Rhoades M.W., Burge C.B., and Bartel D.P. 2003. The microRNAs of Caenorhabditis elegans. Genes Dev. 17: 991.

Lin S.Y., Johnson S.M., Abraham M., Vella M.C., Pasquinelli A., Gamberi C., Gottlieb E., and Slack F.J. 2003. The C. elegans hunchback homolog, hbl-1, controls temporal patterning and is a probable microRNA target. Dev. Cell 4: 639.

Liu Z., Kirch S., and Ambros V. 1995. The Caenorhabditis elegans heterochronic gene pathway controls stage-specific transcription of collagen genes. Development 121: 2471.

Moss E.G., Lee R.C., and Ambros V. 1997. The cold shock domain protein LIN-28 controls developmental timing in $C$. elegans and is regulated by the lin-4 RNA. Cell 88: 637 .

Parrish J.Z., Yang C., Shen B., and Xue D. 2003. CRN-1, a Caenorhabditis elegans FEN-1 homologue, cooperates with CPS-6/EndoG to promote apoptotic DNA degradation. EMBO J. 22: 3451.

Pasquinelli A.E., Reinhart B.J., Slack F., Martindale M.Q., Kuroda M.I., Maller B., Hayward D.C., Ball E.E., Degnan B., Muller P., et al. 2000. Conservation of the sequence and temporal expression of let-7 heterochronic regulatory RNA. Nature 408: 86.

Reinhart B.J., Slack F.J., Basson M., Pasquinelli A.E., Bettinger J.C., Rougvie A.E., Horvitz H.R., and Ruvkun G. 2000. The 21-nucleotide let-7 RNA regulates developmental timing in Caenorhabditis elegans. Nature 403: 901.

Rougvie A.E. and Ambros V. 1995. The heterochronic gene lin29 encodes a zinc finger protein that controls a terminal differentiation event in Caenorhabditis elegans. Development 121: 2491 .

Schulman B.R., Esquela-Kerscher A., and Slack F.J. 2005. Reciprocal expression of lin-41 and the microRNAs let-7 and mir-125 during mouse embryogenesis. Dev. Dyn. 234: 1046.

Sempere L.F., Dubrovsky E.B., Dubrovskaya V.A., Berger E.M., and Ambros V. 2002. The expression of the let-7 small regulatory RNA is controlled by ecdysone during metamorphosis in Drosophila melanogaster. Dev. Biol. 244: 170.

Sempere L.F., Sokol N.S., Dubrovsky E.B., Berger E.M., and Ambros V. 2003. Temporal regulation of microRNA expression in Drosophila melanogaster mediated by hormonal signals and broad-Complex gene activity. Dev. Biol. 259: 9.

Slack F.J., Basson M., Liu Z., Ambros V., Horvitz H.R., and Ruvkun G. 2000. The lin-41 RBCC gene acts in the C. elegans heterochronic pathway between the let-7 regulatory RNA and the LIN-29 transcription factor. Mol. Cell 5: 659.

Sulston J.E. and Horvitz H.R. 1977. Post-embryonic cell lineages of the nematode, Caenorhabditis elegans. Dev. Biol. 56: 110 .

Takamizawa J., Konishi H., Yanagisawa K., Tomida S., Osada H., Endoh H., Harano T., Yatabe Y., Nagino M., Nimura Y., et al. 2004. Reduced expression of the let-7 microRNAs in human lung cancers in association with shortened postoperative survival. Cancer Res. 64: 3753.

Thomson J.M., Newman M., Parker J.S., Morin-Kensicki E.M., Wright T., and Hammond S.M. 2006. Extensive post-transcriptional regulation of microRNAs and its implications for cancer. Genes Dev. 20: 2202.

Wickens M., Bernstein D.S., Kimble J., and Parker R. 2002. A PUF family portrait: 3'UTR regulation as a way of life. Trends Genet. 18: 150.

Wightman B., Ha I., and Ruvkun G. 1993. Posttranscriptional regulation of the heterochronic gene lin-14 by lin- 4 mediates temporal pattern formation in C. elegans. Cell 75: 855. 


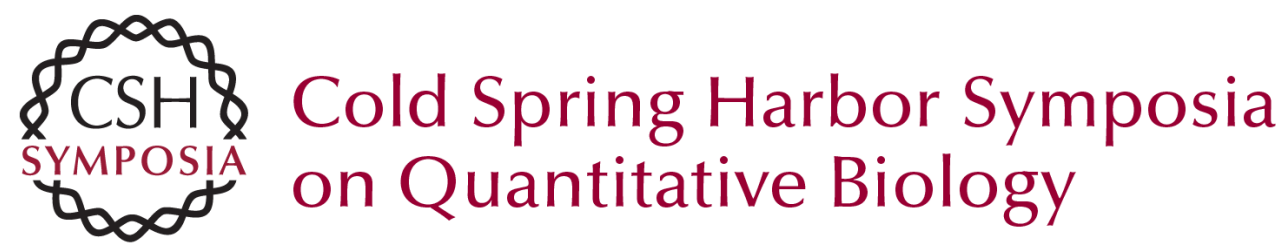

\section{Misexpression of the Caenorhabditis elegans miRNA let-7 Is Sufficient to Drive Developmental Programs}

G.D. HAYES and G. RUVKUN

Cold Spring Harb Symp Quant Biol 2006 71: 21-27

Access the most recent version at doi:10.1101/sqb.2006.71.018

References This article cites 40 articles, 14 of which can be accessed free at: http://symposium.cshlp.org/content/71/21.full.html\#ref-list-1

License

Email Alerting Receive free email alerts when new articles cite this article - sign up in Service the box at the top right corner of the article or click here. 